\title{
EVALUATION OF CYTOCHROME P450-DERIVED EICOSANOIDS IN HUMANS WITH STABLE ATHEROSCLEROTIC CARDIOVASCULAR DISEASE
}

\author{
Katherine N. Theken ${ }^{\mathrm{a}}$, Robert N. Schuck ${ }^{\mathrm{a}}$, Matthew L. Edin ${ }^{\mathrm{e}}$, Bryant Tran ${ }^{\mathrm{a}}$, Kyle Ellis ${ }^{\mathrm{a}}$, \\ Almasa Bass ${ }^{\mathrm{a}}$, Fred B. Lih ${ }^{\mathrm{e}}$, Kenneth B. Tomer ${ }^{\mathrm{e}}$, Samuel M. Poloyac ${ }^{\dagger}$, Michael C. Wu ${ }^{\mathrm{b}}$, Alan \\ L. Hinderliter ${ }^{c, d}$, Darryl C. Zeldine, George A. Stouffer ${ }^{c, d}$, and Craig R. Lee ${ }^{a, d}$ \\ aDivision of Pharmacotherapy and Experimental Therapeutics, Eshelman School of Pharmacy, \\ University of North Carolina, Chapel Hill, NC \\ bDepartment of Biostatistics, Gillings School of Global Public Health, University of North Carolina, \\ Chapel Hill, NC \\ 'Division of Cardiology, School of Medicine, University of North Carolina, Chapel Hill, NC \\ ${ }^{d}$ McAllister Heart Institute, University of North Carolina, Chapel Hill, NC \\ eDivision of Intramural Research, National Institute for Environmental Health Sciences, National \\ Institutes of Health, Research Triangle Park, NC \\ fDepartment of Pharmaceutical Sciences, School of Pharmacy, University of Pittsburgh, \\ Pittsburgh, PA
}

\section{Abstract}

Objective-Preclinical and genetic epidemiologic studies suggest that modulating cytochrome P450 (CYP)-mediated arachidonic acid metabolism may have therapeutic utility in the management of coronary artery disease (CAD). However, predictors of inter-individual variation in CYP-derived eicosanoid metabolites in CAD patients have not been evaluated to date. Therefore, the primary objective was to identify clinical factors that influence CYP epoxygenase, soluble epoxide hydrolase (sEH), and CYP $\omega$-hydroxylase metabolism in patients with established CAD.

Methods-Plasma levels of epoxyeicosatrienoic acids (EETs), dihydroxyeicosatrienoic acids (DHETs), and 20-hydroxyeicosatetraenoic acid (20-HETE) were quantified by HPLC-MS/MS in a population of patients with stable, angiographically-confirmed CAD $(\mathrm{N}=82)$ and healthy volunteers from the local community $(\mathrm{N}=36)$. Predictors of CYP epoxygenase, $\mathrm{sEH}$, and CYP $\omega$ hydroxylase metabolic function were evaluated by regression.

(C) 2012 Elsevier Ireland Ltd. All rights reserved.

Correspondence to: Craig R. Lee, Pharm.D., Ph.D., 2317 Kerr Hall, CB\# 7569, Chapel Hill, NC 27599; Phone: (919) 843-7673; Fax: (919) 962-0644; craig_lee@unc.edu.

Conflicts of Interest

Dr. Zeldin is a co-inventor on U.S. Patent No.6,531,506 B1 (issued March 11, 2003) titled "Inhibition of Epoxide Hydrolases for the Treatment of Hypertension," U.S. Patent No.6,693,130 B2 (issued February 17, 2004) titled "Inhibition of Epoxide Hydrolases for the Treatment of Hypertension," and U.S. Patent No.6,916,843 B1 (issued July 12, 2005) titled "Anti-inflammatory Actions of Cytochrome P450 Epoxygenase-Derived Eicosanoids." No other authors have conflicts of interest to disclose.

Publisher's Disclaimer: This is a PDF file of an unedited manuscript that has been accepted for publication. As a service to our customers we are providing this early version of the manuscript. The manuscript will undergo copyediting, typesetting, and review of the resulting proof before it is published in its final citable form. Please note that during the production process errors may be discovered which could affect the content, and all legal disclaimers that apply to the journal pertain. 
Results-Obesity was significantly associated with low plasma EET levels and 14,15-EET: 14,15-DHET ratios. Age, diabetes, and cigarette smoking also were significantly associated with CYP epoxygenase and sEH metabolic activity, while only renin-angiotensin system inhibitor use was associated with CYP $\omega$-hydroxylase metabolic activity. Compared to healthy volunteers, both obese and non-obese CAD patients had significantly higher plasma EETs $(\mathrm{P}<0.01)$ and epoxide:diol ratios $(\mathrm{P}<0.01)$, whereas no difference in 20-HETE levels was observed $(\mathrm{P}=\mathrm{NS})$.

Conclusions-Collectively, these findings suggest that CYP-mediated eicosanoid metabolism is dysregulated in certain subsets of CAD patients, and demonstrate that biomarkers of CYP epoxygenase and $\mathrm{sEH}$, but not CYP $\omega$-hydroxylase, metabolism are altered in stable CAD patients relative to healthy individuals. Future studies are necessary to determine the therapeutic utility of modulating these pathways in patients with CAD.

\section{Keywords}

Soluble epoxide hydrolase; arachidonic acid; epoxyeicosatrienoic acid; dihydroxyeicosatrienoic acid; 20-hydroxyeicosatetraenoic acid; coronary artery disease

\section{Introduction}

Cardiovascular disease is the leading cause of morbidity and mortality worldwide. Despite advances in diagnosis and treatment, novel therapies are needed to further improve outcomes. Accumulating preclinical and epidemiologic evidence suggests that modulation of cytochrome P450 (CYP)-mediated eicosanoid metabolism may be a viable therapeutic strategy for the management of cardiovascular disease.

The CYP epoxygenases (CYP2J2, CYP2C8) metabolize arachidonic acid to four epoxyeicosatrienoic acid regioisomers (5,6-, 8,9-, 11,12-, 14,15-EET), which possess potent vasodilatory and anti-inflammatory effects [1]. EETs are rapidly hydrolyzed by soluble epoxide hydrolase (sEH) to the corresponding dihydroxyeicosatrienoic acids (DHETs), which are generally less biologically active $[1,2]$. In contrast, $\omega$-hydroxylation of arachidonic acid by CYP4A11 and CYP4F2 produces 20-hydroxyeicosatetraenoic acid (20HETE), a vasoconstrictive and pro-inflammatory eicosanoid [2]. Numerous preclinical studies have demonstrated that potentiation of the CYP epoxygenase pathway, via enhanced EET biosynthesis or inhibition of sEH-mediated EET hydrolysis, and inhibition of 20-HETE biosynthesis are protective in models of cardiovascular disease, including endothelial dysfunction and hypertension, nuclear factor-kappa B dependent vascular inflammation, atherosclerotic lesion development, and ischemia/reperfusion injury [3-8]. Furthermore, functional genetic polymorphisms in the CYP epoxygenases (CYP2J2, CYP2C8), sEH (EPHX2), and CYP $\omega$-hydroxylases (CYP4A11, CYP4F2) have been associated with cardiovascular disease susceptibility in several epidemiologic studies [9-13].

Collectively, this growing body of evidence suggests that CYP-mediated eicosanoid metabolism is integral to the pathogenesis and progression of cardiovascular disease. Consequently, sEH and CYP $\omega$-hydroxylase inhibitors are currently in development $[14,15]$, and these novel therapies are hypothesized to elicit cardiac and vascular protective effects in humans with cardiovascular disease, as observed in preclinical models, via their ability to increase EET and decrease 20-HETE levels, respectively. Despite these advances, very few studies to date have actually quantified CYP-derived eicosanoid metabolite levels in humans. Moreover, the clinical factors associated with inter-individual variation in EET and 20-HETE levels in patients with established cardiovascular disease have not been investigated to date. Advancement of our understanding of the key factors that regulate CYP-mediated eicosanoid metabolism in humans will lay an essential foundation for the design of future studies that seek to evaluate the cardiovascular protective effects of novel 
therapies that increase EET and decrease 20-HETE levels, while also identifying subsets of the population with dysregulated eicosanoid metabolism (i.e., low EET and/or high 20HETE levels) who may be most likely to derive benefit from these novel therapies. Therefore, the primary objective of this study was to identify the key clinical factors associated with inter-individual variation in CYP epoxygenase, $\mathrm{sEH}$, and CYP $\omega$ hydroxylase metabolism in patients with stable atherosclerotic cardiovascular disease. An important secondary objective was to determine whether circulating levels of these metabolic biomarkers significantly differ relative to healthy individuals at low risk for cardiovascular disease.

\section{Methods}

Please see the Online Supplemental Material for an expanded description of the methods.

\section{Study population and protocol}

A cohort of individuals $\$ 65$ years of age with angiographically-confirmed coronary artery disease (CAD), defined as $\mathbf{2 5 0 \%}$ stenosis in at least one major epicardial coronary artery by coronary angiography, were identified in the University of North Carolina (UNC) Cardiac Catheterization Laboratory. A parallel cohort of healthy volunteers $\$ 65$ years of age were identified by advertisement. Individuals with a history of cardiovascular disease, risk factors for CAD (including hypertension, diabetes, cigarette smoking within the previous 6 months, high cholesterol, or body mass index [BMI] $330 \mathrm{~kg} / \mathrm{m}^{2}$ ), or currently taking medication for a chronic medical condition were excluded. After fasting overnight and withholding their morning medications, eligible participants provided informed consent and returned to the clinical research unit for blood sample collection. Participants in the CAD cohort returned $62 \pm 34$ days after their index catheterization and were clinically stable at the time of their study visit. The study was approved by the UNC Biomedical Institutional Review Board.

\section{Quantification of plasma CYP-derived eicosanoids}

Plasma eicosanoid levels were quantified by HLPC-MS/MS, following solid-phase extraction, as described $[12,16]$. Briefly, CYP-derived metabolites of arachidonic acid (14,15-EET, 11,12-EET, 8,9-EET, 14,15-DHET, 11,12-DHET, 8,9-DHET, 5,6-DHET, and 20-HETE) and linoleic acid (9,10- and 12,13-epoxyoctadecenoic acid [9,10-EpOME and 12,13-EpOME] and 9,10- and 12,13-dihydroxyoctadecenoic acid [9,10-DHOME and 12,13DHOME]) were separated by reverse phase HPLC on a 1x150 mm, $5 \mu \mathrm{m}$ Luna C18(2) column (Phenomenex, Torrance, CA) and quantified using a MDS Sciex API 3000 triple quadrupole mass spectrometer (Applied Biosystems, Foster City, CA) with negative mode electrospray ionization and multiple reaction monitoring.

\section{Statistical analysis}

Data are presented as mean \pm standard deviation or median (interquartile range) unless otherwise indicated. Inter-metabolite correlations were determined by Spearman rank correlation to assess redundancy in each biomarker. In the primary cross-sectional analysis, stepwise multiple regression was used to identify clinical factors associated with CYP epoxygenase (sum EETs, sum DHETs), sEH (14,15-EET:14,15-DHET ratio) and CYP $\omega$ hydroxylase (20-HETE) metabolic function in the full CAD cohort. Potential covariates included demographic factors (age, gender, race), indices of CAD severity (presence of multivessel disease, presence of acute coronary syndrome at index catheterization), comorbidities (hypertension, diabetes, cigarette smoking), BMI, and angiotensin converting enzyme (ACE) inhibitor/angiotensin receptor blocker (ARB) use. Covariates with $\mathrm{p}<0.15$ were included in the final model. The secondary analysis, a case-control comparison of each biomarker across the non-obese (BMI $<30 \mathrm{~kg} / \mathrm{m}^{2}$ ) and obese $\left(\mathrm{BMI} \geq 30 \mathrm{~kg} / \mathrm{m}^{2}\right) \mathrm{CAD}$ and 
healthy volunteer cohorts, was completed by regression. Stratified analyses were also completed to assess the potential contribution of confounding factors to the observed differences in each biomarker. The significance level was set at $\mathrm{P}=0.0125(0.05 / 4)$ to account for the impact of multiple statistical tests. At an $a=0.0125$ level, a sample size of 36 per group provided $80 \%$ power to detect a $40 \%$ difference in each biomarker across cohorts, assuming a coefficient of variation of 50\% [12]. All analyses were performed using SAS 9.1.3 (SAS Institute, Cary, NC).

\section{Results \\ Study population}

The population characteristics are shown in Table 1 . The majority of CAD patients had advanced disease, with $25(30.5 \%)$ presenting with an acute coronary syndrome at their index catheterization and $52(63.4 \%)$ diagnosed with multivessel disease. More than half of the $\mathrm{CAD}$ patients were obese. Medication utilization rates were consistent with current clinical practice guidelines.

\section{Correlation between biomarkers of CYP epoxygenase, sEH and CYP w-hydroxylase function}

The relative plasma levels each metabolite are reported in Supplemental Figure 1. The EETs circulated at the lowest concentrations, while the linoleic acid metabolites (EpOMEs, DHOMEs) were present at much higher concentrations. All epoxide metabolites were highly positively correlated, as were the diols (Supplemental Figure 1A). 20-HETE was positively correlated with the diols, but not the epoxides. The 14,15-EET:14,15-DHET ratio positively correlated with the 9,10-EpOME:9,10-DHOME $\left(\mathrm{r}_{\mathrm{S}}=0.81\right.$; $\left.\mathrm{P}<0.001\right)$ and 12,13-EpOME: $12,13-\mathrm{DHOME}\left(\mathrm{r}_{\mathrm{S}}=0.86, \mathrm{P}<0.001\right)$ ratios, which were also significantly correlated $\left(\mathrm{r}_{\mathrm{S}}=0.88\right.$; $\mathrm{P}<0.001$ ). Similar results were observed in healthy volunteers (Supplemental Figure 1B). Due to the high degree of inter-metabolite correlation, sum EETs and sum DHETs, the 14,15-EET:14,15-DHET ratio, and 20-HETE levels were used as biomarkers of CYP epoxygenase, $\mathrm{sEH}$, and CYP $\omega$-hydroxylase metabolic activity, respectively, to minimize redundancy in the analysis.

\section{Cross-sectional analysis of clinical factors associated with biomarkers of CYP epoxygenase, sEH and CYP $\omega$-hydroxylase function in stable CAD patients}

Several clinical covariates were associated with CYP epoxygenase pathway function. Plasma sum EETs were negatively associated with age and BMI (Table 2), such that sum EETs were significantly lower in obese $(0.25[0.17] \mathrm{ng} / \mathrm{mL})$, compared to non-obese CAD patients $(0.34$ [0.32] ng/mL; $\mathrm{P}<0.001$; Figure $1 \mathrm{~A})$. Plasma sum DHETs were negatively associated with presence of diabetes and positively associated with smoking status (Table 2). No relationship with obesity was observed (Figure 1B).

Consistent with the relationships observed for EETs and DHETs, the 14,15-EET:14,15DHET ratio was negatively associated with BMI and smoking status (Table 2). Obese CAD patients had a significantly lower 14,15 -EET:14,15-DHET ratios ( 0.23 [0.49]), compared to non-obese patients $(0.52$ [0.59]; $\mathrm{P}=0.011$; Figure 1C). Similarly, smokers tended to have lower 14,15-EET:14,15-DHET ratios (0.23 [0.35]), compared to non-smokers (0.42 [0.65]; $\mathrm{P}=0.061$ ). In contrast, no difference was observed in diabetics compared to non-diabetics $(\mathrm{P}=0.500)$.

Plasma 20-HETE levels were negatively associated with renin-angiotensin system inhibitor use (Table 2), such that patients treated with an ACE inhibitor or ARB had significantly lower plasma 20-HETE levels $(1.40[0.71] \mathrm{ng} / \mathrm{mL})$ compared to patients not treated with 
these medications (1.68 [1.18] ng/mL; $\mathrm{P}=0.004)$. Since only five CAD patients received an $\mathrm{ARB}$, this association was driven by ACE inhibitor use (data not shown). However, no significant association with age, BMI, diabetes or smoking was observed, and 20-HETE levels were not significantly different between obese $(1.41[0.66] \mathrm{ng} / \mathrm{mL})$ and non-obese patients (1.60 [0.97] ng/mL; P=0.249; Figure 1D).

No significant relationships were observed between biomarkers of CYP-mediated eicosanoid metabolism and either gender, race, multivessel disease, or a recent acute coronary syndrome (Supplemental Table 1).

\section{Case-control comparison}

Because obesity was associated with lower EETs and epoxide:diol ratios (Figure 1), the CAD cohort was stratified by obesity status prior to comparison with the healthy volunteer cohort. Compared to the healthy volunteers, the CAD patients were significantly older, had higher blood pressure and serum triglycerides, and had lower HDL, LDL, and total cholesterol levels (Table 1). The latter observation is likely due to treatment with lipidlowering therapies.

Both non-obese and obese CAD patients had significantly higher sum EETs compared to healthy volunteers (non-obese CAD: $\mathrm{P}<0.001$; obese CAD: $\mathrm{P}=0.007$; Figure $1 \mathrm{~A}$ ). These relationships remained significant after adjusting for age, gender, and race $(\mathrm{P}<0.001$ for both). CAD patients tended to have lower sum DHETs compared to healthy volunteers, but this difference was not statistically significant (non-obese CAD: $\mathrm{P}=0.093$; obese CAD: $\mathrm{P}=0.037$; Figure 1B). Similar differences were observed with the individual regioisomers (Supplemental Table 2).

The epoxide:diol ratios were markedly different across cohorts, such that the 14,15-EET: 14,15-DHET ratio was significantly higher in both subsets of CAD patients compared to healthy volunteers (non-obese CAD: $\mathrm{P}<0.001$; obese $\mathrm{CAD}$ : $\mathrm{P}=0.002$; Figure $1 \mathrm{C}$ ). Similar results were observed for the EpOME:DHOME ratios (Supplemental Table 2). These relationships remained statistically significant when adjusted for age, gender, and race $(\mathrm{P}<0.001$ for all). Similar differences across the $\mathrm{CAD}$ and healthy volunteer cohorts were also observed after stratifying by gender, smoking status, and diabetes status (Supplemental Figure 2; $\mathrm{P}<0.0125$ for all comparisons).

In contrast, no differences in plasma 20-HETE levels were observed between CAD patients and healthy volunteers (non-obese CAD: $\mathrm{P}=0.508$; obese $\mathrm{CAD}$ : $\mathrm{P}=0.600$; Figure $1 \mathrm{D}$ ). After stratifying by ACE inhibitor/ARB use, 20-HETE levels tended to be higher in CAD patients not treated with a renin-angiotensin system inhibitor $(1.68[1.18] \mathrm{ng} / \mathrm{mL})$ compared to healthy volunteers $(1.48[0.70] \mathrm{ng} / \mathrm{mL})$; however, this difference was not statistically significant (unadjusted: $\mathrm{P}=0.086$; adjusted for age, gender, race, and BMI: $\mathrm{P}=0.022$ ).

\section{Discussion}

Preclinical and genetic epidemiologic studies suggest that increasing EET and/or decreasing 20-HETE levels may have utility as a cardiovascular protective therapeutic strategy in patients with cardiovascular disease. Despite these advances, few studies have actually quantified CYP-derived eicosanoid metabolite levels in humans. To our knowledge, this is the first study to (1) identify the key clinical factors, most notably obesity, associated with inter-individual variation in CYP-mediated eicosanoid metabolism in patients with stable $\mathrm{CAD}$, and (2) demonstrate that circulating biomarkers of CYP epoxygenase and $\mathrm{sEH}$, but not CYP $\omega$-hydroxylase, metabolic function are altered in patients with established atherosclerotic cardiovascular disease relative to individuals at low risk for cardiovascular 
disease. Collectively, these findings provide novel insight into which subsets of the population exhibit dysregulated CYP-mediated eicosanoid metabolism, and lay a foundation for the rational design of future studies that aim to evaluate the cardiovascular protective effects of novel therapies that directly modulate CYP-derived EET and 20-HETE levels.

Obesity was the strongest predictor of CYP epoxygenase metabolic function identified. The inverse relationship between BMI and both plasma EET levels and 14,15-EET:14,15-DHET ratios suggests that CYP epoxygenase activity is suppressed and sEH metabolic activity is induced, respectively, in obese individuals. These findings are consistent with the suppression of CYP epoxygenase and induction of sEH expression and metabolic activity in preclinical models of obesity [17,18]; however, the underlying mechanisms remain unclear and largely unexplored. We recently reported that hepatic CYP epoxygenase expression and metabolic activity is significantly suppressed in high-fat diet-fed mice. This was independent of hypercholesterolemia and insulin resistance and was reversed by ACE inhibition, but not angiotensin type 1 (AT1) receptor inhibition, suggesting that angiotensin type 2 (AT2) and/or bradykinin B2 receptor signaling regulates obesity-induced changes in CYP epoxygenase function [18].

Several other clinical factors were associated with CYP epoxygenase and sEH metabolic function, albeit to a lesser degree. Age was inversely associated with plasma EET levels, consistent with a prior study demonstrating a decline in renal CYP epoxygenase activity in aging rats [19]. The inverse relationship between diabetes and DHET levels, but not the 14,15-EET:14,15-DHET ratio, suggests lower CYP epoxygenase activity, consistent with suppression of CYP2C expression in a rat model of diabetes [20]. Cigarette smoking was associated with higher DHETs and lower epoxide:diol ratios, suggesting that smoking increases sEH expression or metabolic activity. This is consistent with higher vascular sEH expression observed in mice chronically exposed to tobacco smoke [21]. Interestingly, cigarette smoking also modifies the risk of developing CAD in individuals who carry functional genetic variants in $E P H X 2$ [12], $C Y P 2 C 8$ [11], and $C Y P 2 J 2$ [22], such that variant allele carriers who smoke have the highest risk. Our findings suggest that these geneenvironment interactions may be mediated in part by an upregulation of sEH expression and EET hydrolysis in cigarette smokers. However, studies evaluating the direct effect of cigarette smoke exposure on $\mathrm{sEH}$ function in humans are needed to confirm this hypothesis. Although the specific molecular mechanisms remain unclear and require further study, we observed that dysregulation of CYP epoxygenase pathway function exists in the presence of pathologic stimuli known to drive the progression of cardiovascular dysfunction (i.e., obesity, advancing age, diabetes, cigarette smoking), which is collectively consistent with existing evidence in preclinical models [1].

Of the clinical factors evaluated, only renin-angiotensin system inhibitor use was associated with CYP $\omega$-hydroxylase metabolic function. This observation is consistent with a previous report demonstrating significantly higher plasma 20-HETE levels, a potent vasoconstrictor, in patients with renovascular disease, a condition characterized by activation of the reninangiotensin system, and a positive correlation between 20-HETE levels and plasma renin activity [23]. In preclinical studies, angiotensin II stimulates 20-HETE release in renal microvessels via an AT2 receptor-dependent mechanism [24]. Furthermore, ACE, but not AT1 receptor, inhibition reverses high fat diet-induced upregulation of renal CYP $\omega$ hydroxylase activity, further implicating AT2 receptor signaling as a key regulator of 20HETE biosynthesis [18]. In contrast to prior studies showing a correlation between urinary 20-HETE levels and BMI [25,26], we observed no relationship between plasma 20-HETE and obesity. Several factors may account for these differences. First, urinary 20-HETE levels may reflect local production in the kidney rather than systemic levels. As a result, the clinical factors that influence urinary 20-HETE excretion may differ from those associated 
with plasma levels. Indeed, we recently reported that high fat diet administration upregulates CYP $\omega$-hydroxylase activity in kidney, but not liver [18]. Second, our study population was composed of stable CAD patients with multiple comorbidities, while previous studies have exclusively enrolled untreated individuals with hypertension or metabolic syndrome. Consequently, the effects of drug therapy may have masked other factors that influence plasma 20-HETE levels in CAD patients. Further study is necessary to elucidate the mechanisms underlying functional interactions between obesity, the reninangiotensin system and CYP $\omega$-hydroxylase metabolism.

Interestingly, we observed that the epoxide:diol ratios were markedly higher in CAD patients compared to healthy volunteers, in all subsets of the population, indicating significantly lower apparent sEH metabolic activity in the presence of stable atherosclerotic cardiovascular disease. Consistent with these findings, CAD patients also had higher EETs and tended to have lower DHETs compared to healthy volunteers. It is well-established that EETs possess potent vasodilatory and anti-inflammatory properties, and sEH inhibition elicits a myriad of cardiovascular protective effects in preclinical models via increasing EET levels $[1,4,6,7]$. In humans, carriers of a genetic polymorphism associated with higher sEH activity $(E P H X 2 \mathrm{~K} 55 \mathrm{R})$ had a greater risk of incident CAD [12] and enhanced vascular resistance [27]. Consequently, the observed suppression of sEH metabolism in patients with stable $\mathrm{CAD}$, relative to the healthy volunteers, would be hypothesized to be cardioprotective and serve as a compensatory response to the presence of advanced cardiovascular disease.

Although the mechanisms underlying a compensatory suppression of sEH remain unclear, a prior report demonstrated that although the gene encoding SEH (EPHX2) was a susceptibility gene for heart failure in preclinical models, humans with established heart failure exhibited 60\% lower cardiac sEH expression compared to healthy controls [28]. These data suggest that transcriptional down-regulation of sEH, to increase EET levels, may be an adaptive mechanism in human cardiovascular disease [28]. Our findings provide further support for this hypothesis. Indeed, a compensatory up-regulation of other "protective" mediators, most notably the natriuretic peptides, is well known to occur in the presence of stable and unstable cardiovascular disease [29]. In addition, reactive oxygen species inhibit sEH metabolic activity in vitro [30], suggesting that enhanced oxidative stress in the presence of established CAD may also contribute to the observed suppression of sEH metabolic activity. Due to the cardioprotective effects of EETs in preclinical models of myocardial ischemia/reperfusion injury [1,7], subsequent studies are warranted to determine whether a compensatory suppression of sEH metabolism also occurs in unstable CAD patients experiencing an acute coronary syndrome. Importantly, sEH inhibitors have entered clinical trials for the treatment of cardiovascular and metabolic disease [14]. Interventional studies with these agents will ultimately be necessary to determine whether pharmacologic suppression of sEH to further increase EET levels will elicit cardiovascular protective effects and improve outcomes in patients with established cardiovascular disease. Our findings suggest that certain subsets of the population, most notably obese individuals, may be "resistant" to a compensatory suppression of sEH metabolic activity, and thus may be more likely to derive therapeutic benefit from $\mathrm{sEH}$ inhibition. This hypothesis, however, requires prospective evaluation.

There are several limitations to our analysis. Due to the cross-sectional and observational design, we are unable to establish a cause-effect relationship between the observed relationships, most notably the apparent suppression of sEH metabolism in the presence of CAD. In addition, our analysis compared a well-treated population of patients with advanced cardiovascular disease and multiple comorbidities to healthy individuals with no risk factors for cardiovascular disease. Thus, multiple potential confounding factors may have influenced the differences in epoxide:diol ratios. For example, we cannot determine 
whether the observed differences are due to the presence of atherosclerotic disease, the presence of comorbidities/cardiovascular risk factors, or a consequence of drug therapy. Although the observed differences across cohorts persisted after adjusting for and stratifying by demographic and clinical factors, nearly all the CAD patients were treated with aspirin, statins, and beta-blockers. Thus, we were unable to ascertain the effects of drug therapy on eicosanoid levels. Since the effects of these established therapies on circulating CYPderived eicosanoid levels, and specifically sEH expression and metabolic activity, in humans are unknown, further studies are necessary to quantify these effects. Finally, although we adjusted the significance level to account for the impact of multiple statistical tests, replication in an independent cohort is necessary to validate our results.

Despite these limitations, our study is the first to identify clinical factors associated with inter-individual variation in CYP-mediated eicosanoid metabolism in patients with established cardiovascular disease. Pharmacologic agents that increase EETs (sEH inhibitors) or decrease 20-HETE (CYP $\omega$-hydroxylase inhibitors) are in development and may represent a novel therapeutic strategy for the secondary prevention of cardiovascular events in patients with established CAD. Since such novel therapies would most likely be used in addition to the current standards of care (i.e., statins, aspirin, beta-blockers), our study was designed to identify the clinical factors associated with biomarkers of CYP epoxygenase, $\mathrm{sEH}$, and CYP $\omega$-hydroxylase metabolism in a population of stable, welltreated patients with established CAD. The reported results (1) provide novel insight into which subsets of the population exhibit dysregulated CYP-mediated eicosanoid metabolism; and, (2) lay a foundation for future clinical research in this area, including the rational design of interventional studies that aim to evaluate the cardiovascular protective effects of increasing CYP-derived EET and decreasing CYP-derived 20-HETE levels, in addition to current standards of care, as a novel therapeutic strategy for patients with established CAD. Most notably, our findings suggest that targeted evaluation of the cardiovascular protective effects of sEH inhibition in obese CAD patients may be warranted.

\section{Conclusions}

Our cross-sectional analysis identified the primary clinical factors associated with CYP epoxygenase/sEH (obesity, advancing age, diabetes, cigarette smoking) and CYP $\omega$ hydroxylase (renin-angiotensin inhibitor use) metabolism in a population of patients with established CAD. In addition, the case-control analysis demonstrated for the first time that sEH metabolic activity is suppressed in stable CAD patients relative to healthy individuals. These findings lay a foundation for future studies that aim to elucidate the specific mechanisms underlying the impact of each clinical factor on CYP-mediated eicosanoid metabolism, and define the therapeutic utility of modulating CYP-derived EET and 20HETE levels in patients with cardiovascular disease.

\section{Supplementary Material}

Refer to Web version on PubMed Central for supplementary material.

\section{Acknowledgments}

The authors gratefully acknowledge the UNC Clinical and Translational Research Center staff for their assistance.

Funding

This publication was made possible by a predoctoral fellowship from the American Foundation for Pharmaceutical Education to Dr. Theken, a predoctoral training program in Integrative Vascular Biology supported by the National Institutes of Health National Heart, Lung, and Blood Institute (T32 HL069768) to Dr. Schuck, a Beginning Grantin-Aid from the American Heart Association and a pilot grant from the North Carolina Translational and Clinical 
Sciences Institute to Dr. Lee, funds from the Intramural Research Program of the NIH/NIEHS to Dr. Tomer (Z01 ES050167) and Dr. Zeldin (Z01 ES025034), and in part by grants M01RR00046 and UL1RR025747 from the NIH/ NCRR. Its contents are solely the responsibility of the authors and do not necessarily represent the official views of the NIH.

\section{References}

1. Deng Y, Theken KN, Lee CR. Cytochrome P450 epoxygenases, soluble epoxide hydrolase, and the regulation of cardiovascular inflammation. J Mol Cell Cardiol. 2010; 48:331-341. [PubMed: 19891972]

2. Roman RJ. P-450 metabolites of arachidonic acid in the control of cardiovascular function. Physiol Rev. 2002; 82:131-185. [PubMed: 11773611]

3. Lee CR, Imig JD, Edin ML, et al. Endothelial expression of human cytochrome P450 epoxygenases lowers blood pressure and attenuates hypertension-induced renal injury in mice. FASEB J. 2010; 24:3770-3781. [PubMed: 20495177]

4. Deng Y, Edin ML, Theken KN, et al. Endothelial CYP epoxygenase overexpression and soluble epoxide hydrolase disruption attenuate acute vascular inflammatory responses in mice. FASEB J. 2011; 25:703-713. [PubMed: 21059750]

5. Wu CC, Cheng J, Zhang FF, et al. Androgen-dependent hypertension is mediated by 20hydroxy-5,8,11,14-eicosatetraenoic acid-induced vascular dysfunction: role of inhibitor of kappaB Kinase. Hypertension. 2011; 57:788-794. [PubMed: 21321301]

6. Zhang LN, Vincelette J, Cheng Y, et al. Inhibition of soluble epoxide hydrolase attenuated atherosclerosis, abdominal aortic aneurysm formation, and dyslipidemia. Arterioscler Thromb Vasc Biol. 2009; 29:1265-1270. [PubMed: 19667112]

7. Seubert JM, Sinal CJ, Graves J, et al. Role of soluble epoxide hydrolase in postischemic recovery of heart contractile function. Circ Res. 2006; 99:442-450. [PubMed: 16857962]

8. Nithipatikom K, Gross ER, Endsley MP, et al. Inhibition of cytochrome P450omega-hydroxylase: a novel endogenous cardioprotective pathway. Circ Res. 2004; 95:e65-71. [PubMed: 15388642]

9. Fava C, Montagnana M, Almgren P, et al. The V433M variant of the CYP4F2 is associated with ischemic stroke in male Swedes beyond its effect on blood pressure. Hypertension. 2008; 52:373380. [PubMed: 18574070]

10. Gainer JV, Bellamine A, Dawson EP, et al. Functional variant of CYP4A11 20hydroxyeicosatetraenoic acid synthase is associated with essential hypertension. Circulation. 2005; 111:63-69. [PubMed: 15611369]

11. Lee CR, North KE, Bray MS, et al. CYP2J2 and CYP2C8 polymorphisms and coronary heart disease risk: the Atherosclerosis Risk in Communities (ARIC) study. Pharmacogenet Genomics. 2007; 17:349-358. [PubMed: 17429317]

12. Lee CR, North KE, Bray MS, et al. Genetic variation in soluble epoxide hydrolase (EPHX2) and risk of coronary heart disease: The Atherosclerosis Risk in Communities (ARIC) study. Hum Mol Genet. 2006; 15:1640-1649. [PubMed: 16595607]

13. Theken KN, Lee CR. Genetic variation in the cytochrome P450 epoxygenase pathway and cardiovascular disease risk. Pharmacogenomics. 2007; 8:1369-1383. [PubMed: 17979511]

14. Chen D, Whitcomb R, Macintyre E, et al. Pharmacokinetics and pharmacodynamics of AR9281, an inhibitor of soluble epoxide hydrolase, in single- and multiple-dose studies in healthy human subjects. J Clin Pharmacol. 2011 [epub ahead of print, March 21, 2011].

15. Williams JM, Murphy S, Burke M, et al. 20-hydroxyeicosatetraeonic acid: a new target for the treatment of hypertension. J Cardiovasc Pharmacol. 2010; 56:336-344. [PubMed: 20930591]

16. Edin ML, Wang Z, Bradbury JA, et al. Endothelial expression of human cytochrome P450 epoxygenase CYP2C8 increases susceptibility to ischemia-reperfusion injury in isolated mouse heart. FASEB J. 2011; 25:3436-3447. [PubMed: 21697548]

17. Zhao X, Dey A, Romanko OP, et al. Decreased epoxygenase and increased epoxide hydrolase expression in the mesenteric artery of obese Zucker rats. Am J Physiol Regul Integr Comp Physiol. 2005; 288:R188-196. [PubMed: 15345471] 
18. Theken KN, Deng Y, Schuck RN, et al. Enalapril reverses high fat diet-induced alterations in cytochrome P450-mediated eicosanoid metabolism. Am J Physiol Endocrinol Metab. 2012; 302:E500-E509. [PubMed: 22185841]

19. Omata K, Abraham NG, Escalante B, et al. Age-related changes in renal cytochrome P-450 arachidonic acid metabolism in spontaneously hypertensive rats. Am J Physiol. 1992; 262:F8-16. [PubMed: 1733300]

20. Shimojo N, Ishizaki T, Imaoka S, et al. Changes in amounts of cytochrome P450 isozymes and levels of catalytic activities in hepatic and renal microsomes of rats with streptozocin-induced diabetes. Biochem Pharmacol. 1993; 46:621-627. [PubMed: 8363636]

21. Maresh JG, Xu H, Jiang N, et al. Tobacco smoke dysregulates endothelial vasoregulatory transcripts in vivo. Physiol Genomics. 2005; 21:308-313. [PubMed: 15728332]

22. Liu PY, Li YH, Chao TH, et al. Synergistic effect of cytochrome P450 epoxygenase CYP2J2*7 polymorphism with smoking on the onset of premature myocardial infarction. Atherosclerosis. 2007; 195:199-206. [PubMed: 17126841]

23. Minuz P, Jiang H, Fava C, et al. Altered release of cytochrome p450 metabolites of arachidonic acid in renovascular disease. Hypertension. 2008; 51:1379-1385. [PubMed: 18378855]

24. Croft KD, McGiff JC, Sanchez-Mendoza A, et al. Angiotensin II releases 20-HETE from rat renal microvessels. Am J Physiol Renal Physiol. 2000; 279:F544-551. [PubMed: 10966934]

25. Laffer CL, Laniado-Schwartzman M, Nasjletti A, et al. 20-HETE and circulating insulin in essential hypertension with obesity. Hypertension. 2004; 43:388-392. [PubMed: 14707157]

26. Ward NC, Rivera J, Hodgson J, et al. Urinary 20-hydroxyeicosatetraenoic acid is associated with endothelial dysfunction in humans. Circulation. 2004; 110:438-443. [PubMed: 15262846]

27. Lee CR, Pretorius M, Schuck RN, et al. Genetic variation in soluble epoxide hydrolase (EPHX2) is associated with forearm vasodilator responses in humans. Hypertension. 2011; 57:116-122. [PubMed: 21098312]

28. Monti J, Fischer J, Paskas S, et al. Soluble epoxide hydrolase is a susceptibility factor for heart failure in a rat model of human disease. Nat Genet. 2008; 40:529-537. [PubMed: 18443590]

29. Di Angelantonio E, Chowdhury R, Sarwar N, et al. B-type natriuretic peptides and cardiovascular risk: systematic review and meta-analysis of 40 prospective studies. Circulation. 2009; 120:21772187. [PubMed: 19917883]

30. Oguro A, Fujita N, Imaoka S. Regulation of soluble epoxide hydrolase (sEH) in mice with diabetes: high glucose suppresses sEH expression. Drug Metab Pharmacokinet. 2009; 24:438-445. [PubMed: 19881255] 


\section{Highlights}

- We evaluated clinical factors that influence CYP-derived eicosanoids in CAD patients.

- Obesity and advancing age were associated with lower EET levels.

- Obesity and cigarette smoking were associated with lower epoxide:diol ratios.

- Renin-angiotensin system inhibitor use was associated with lower 20-HETE levels.

- CAD patients exhibited lower sEH metabolic activity relative to healthy individuals. 
Sum EETs

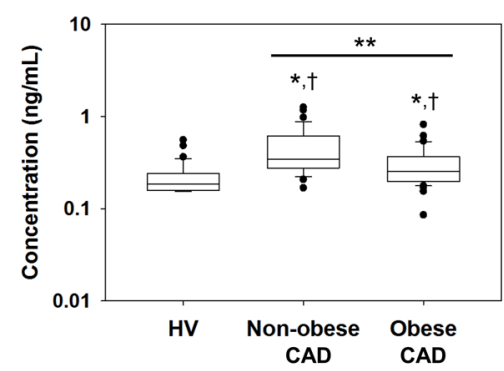

C

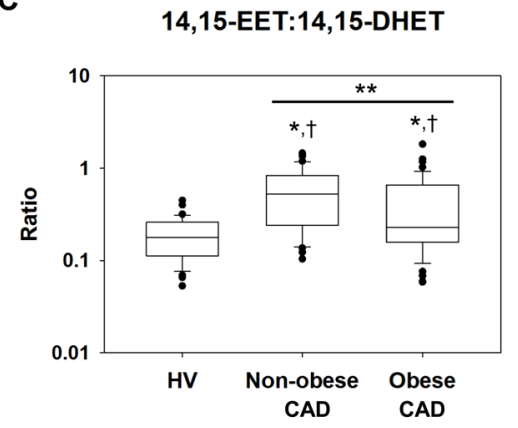

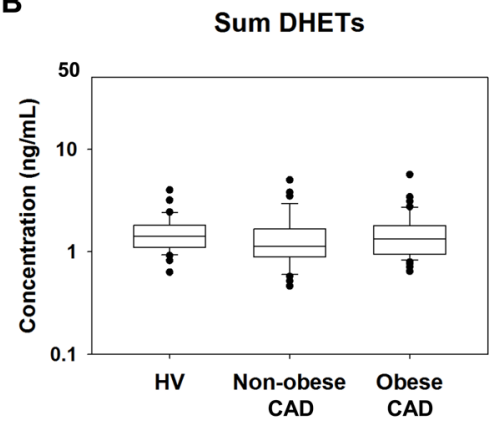

D

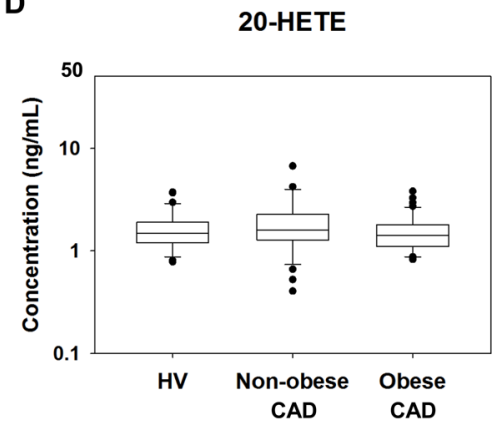

Figure 1.

Distribution of plasma (A) sum EETs, (B) sum DHETs, (C) 14,15-EET:14,15-DHET, and (D) 20-HETE in healthy volunteers ( $\mathrm{HV}, \mathrm{n}=36)$, non-obese CAD patients $(\mathrm{n}=36)$, and obese CAD patients $(n=46)$ plotted on a $\log _{10}$-scale. The line within the box defines the median, the ends of the boxes define the $25^{\text {th }}$ and $75^{\text {th }}$ percentiles, the error bars define the $10^{\text {th }}$ and $90^{\text {th }}$ percentiles, and the individual points identify values outside the $10^{\text {th }}$ and $90^{\text {th }}$ percentiles. *Unadjusted $\mathrm{P}<0.0125$ versus $\mathrm{HV}$. ${ }^{\dagger} \mathrm{P}<0.0125$ versus $\mathrm{HV}$ after adjusting for age, gender, and race. ${ }^{*} * \mathrm{P}<0.0125$ for the obese $\mathrm{CAD}$ versus non-obese CAD comparison. 


\section{Table 1}

Characteristics of the full CAD cohort, non-obese (body mass index $30 \mathrm{~kg} / \mathrm{m}^{2}$ ) and obese (body mass index $30 \mathrm{~kg} / \mathrm{m}^{2}$ ) subsets of the CAD cohort, and healthy volunteer cohort.

\begin{tabular}{|c|c|c|c|c|}
\hline \multirow[t]{2}{*}{ Characteristic } & \multicolumn{3}{|c|}{ Coronary Artery Disease } & \multirow[t]{2}{*}{ Healthy Volunteer } \\
\hline & All & Non-obese & Obese & \\
\hline $\mathrm{N}$ & 82 & 36 & 46 & 36 \\
\hline Age (years) & $54.8 \pm 7.2^{*}$ & $55.4 \pm 6.0^{*}$ & $54.3 \pm 8.7^{*}$ & $50.0 \pm 7.3$ \\
\hline Women $(\%)$ & $31(37.8 \%)$ & $16(44.4 \%)$ & $15(32.6 \%)^{*}$ & $20(55.6 \%)$ \\
\hline African-American (\%) & $14(17.1 \%)$ & $5(13.9 \%)$ & $9(19.6 \%)$ & $6(16.7 \%)$ \\
\hline Body mass index $\left(\mathrm{kg} / \mathrm{m}^{2}\right)$ & $30.6 \pm 5.9^{*}$ & $25.2 \pm 3.2$ & $34.8 \pm 3.6^{*}$ & $25.7 \pm 2.4$ \\
\hline$<25 \mathrm{~kg} / \mathrm{m}^{2}$ & $16(19.5 \%)$ & $16(44.4 \%)$ & $0(0 \%)$ & $12(33.3 \%)$ \\
\hline $25-29.9 \mathrm{~kg} / \mathrm{m}^{2}$ & $20(24.4 \%)$ & $20(55.6 \%)$ & $0(0 \%)$ & $24(66.7 \%)$ \\
\hline$\geq 30 \mathrm{~kg} / \mathrm{m}^{2}$ & $46(56.1 \%)$ & $0(0 \%)$ & $46(100 \%)$ & $0(0 \%)^{\dagger}$ \\
\hline Current smoker $(\%)$ & $22(26.8 \%)$ & $11(30.6 \%)$ & $11(23.9 \%)$ & $0(0 \%)^{\dagger}$ \\
\hline Diabetes (\%) & $22(26.8 \%)$ & $10(27.8 \%)$ & $12(26.1 \%)$ & $0(0 \%)^{\dagger}$ \\
\hline Hypertension (\%) & $63(76.8 \%)$ & $28(77.8 \%)$ & $35(76.1 \%)$ & $0(0 \%)^{\dagger}$ \\
\hline Systolic blood pressure (mmHg) & $135 \pm 17^{*}$ & $136 \pm 15^{*}$ & $133 \pm 18^{*}$ & $122 \pm 13$ \\
\hline Diastolic blood pressure (mmHg) & $80 \pm 10^{*}$ & $80 \pm 11^{*}$ & $80 \pm 10^{*}$ & $75 \pm 8$ \\
\hline Total cholesterol (mmol/L) & $4.0(1.2)^{*}$ & $4.0(1.2)^{*}$ & $4.0(1.5)^{*}$ & $4.9(0.7)$ \\
\hline LDL cholesterol (mmol/L) & $2.2(1.1)^{*}$ & $2.0(0.7)^{*}$ & $2.3(1.3)^{*}$ & $3.0(0.7)$ \\
\hline HDL cholesterol (mmol/L) & $1.2(0.4)^{*}$ & $1.2(0.4)^{*}$ & $1.1(0.4)^{*}$ & $1.7(0.5)$ \\
\hline Triglycerides (mmol/L) & $1.2(0.9)^{*}$ & $1.2(0.9)^{*}$ & $1.2(0.9)^{*}$ & $0.8(0.4)$ \\
\hline Total: HDL cholesterol (ratio) & $3.4(1.7)$ & $3.2(1.2)$ & $3.6(1.7)^{*}$ & $3.1(1.1)$ \\
\hline ACE inhibitor or ARB use (\%) & $50(61.0 \%)$ & $19(52.8 \%)$ & $31(67.4 \%)$ & $0(0 \%)^{\dagger}$ \\
\hline Beta-blocker use (\%) & $69(84.2 \%)$ & $31(86.1 \%)$ & $38(82.6 \%)$ & $0(0 \%)^{\dagger}$ \\
\hline Statin use (\%) & $76(92.7 \%)$ & $34(94.4 \%)$ & $42(91.3 \%)$ & $0(0 \%)^{\dagger}$ \\
\hline Fibrate use (\%) & $1(1.2 \%)$ & $0(0 \%)$ & $1(2.2 \%)$ & $0(0 \%)^{\dagger}$ \\
\hline Aspirin use (\%) & $79(96.3 \%)$ & $35(97.2 \%)$ & $44(95.7 \%)$ & $0(0 \%)^{\dagger}$ \\
\hline Clopidogrel use (\%) & $65(79.3 \%)$ & $26(72.2 \%)$ & $39(84.8 \%)$ & $0(0 \%)^{\dagger}$ \\
\hline
\end{tabular}

$\mathrm{ACE}=$ angiotensin converting enzyme; $\mathrm{ARB}=$ angiotensin receptor blocker; $\mathrm{CAD}=$ coronary artery disease; $\mathrm{HDL}=$ high density lipoprotein; $\mathrm{LDL}=$ low density lipoprotein.

Data presented as mean \pm standard deviation, median (interquartile range) or count (proportion).

* $\mathrm{P}<0.05$ versus healthy volunteers.

Individuals with a history of cardiovascular disease, risk factors for CAD (hypertension, diabetes, cigarette smoking within the previous 6 months, high cholesterol, or body mass index $\geq 30 \mathrm{~kg} / \mathrm{m}^{2}$ ), or currently taking medication for a chronic medical condition were excluded from the healthy volunteer cohort. 
Table 2

Clinical factors associated with biomarkers of CYP epoxygenase, sEH and CYP $\omega$-hydroxylase function in CAD patients.

\begin{tabular}{lcccc}
\hline & Parameter Estimate & SE & Partial R $^{2}$ & p \\
\hline $\begin{array}{l}\text { Sum EETS } \\
\text { Body mass index }\end{array}$ & -0.036 & 0.009 & 0.122 & 0.001 \\
Age & -0.014 & 0.007 & 0.048 & 0.037 \\
Sum DHETS & & & & \\
Smoking & 0.273 & 0.121 & 0.051 & 0.041 \\
Diabetes & -0.255 & 0.121 & 0.051 & 0.038 \\
14,15-EET:14,15-DHET & & & & \\
Smoking & -0.531 & 0.213 & 0.043 & 0.061 \\
Body mass index & -0.038 & 0.016 & 0.041 & 0.064 \\
20-HETE & & & & \\
ACEI/ARB use & -0.320 & 0.109 & 0.098 & 0.004 \\
\hline
\end{tabular}

$\mathrm{ACEI}=$ angiotensin converting enzyme inhibitor; $\mathrm{ARB}=$ angiotensin receptor blocker.

Sum EETs, sum DHETs, 20-HETE, and 14,15-EET:14,15-DHET ratio were log-transformed prior to analysis. 